\title{
Planejamento de experimentos em blocos aplicado às propriedades mecânicas de arames de aço para molas
}

\author{
Cristie Diego Pimenta ${ }^{a *}$, Messias Borges Silva ${ }^{\mathrm{b}}$, Rosinei Batista Ribeiroc, \\ Fernando Antonio Elias Claro ${ }^{\mathrm{d}}$ \\ a*pimentadiego@yahoo.com.br, FEG-UNESP, Brasil \\ ${ }^{b}$ messias.silva@feg.unesp.br, FEG-UNESP, Brasil \\ rosinei1971@gmail.com, FATEA, Brasil \\ dfernandoclaro@uol.com.br, UNITAU, Brasil
}

\begin{abstract}
Resumo
0 objetivo deste artigo é mostrar a aplicação das metodologias planejamento de experimentos com análise em blocos e regressão linear múltipla aplicadas ao estudo de um processo de tratamento térmico siderúrgico com múltiplas respostas. Por meio deste estudo buscou-se projetar modelos estatísticos que permitissem prever os resultados das propriedades mecânicas em arames de aço SAE 9254 trefilados, com diâmetros de 2,00 mm e 6,50 mm, após processo de têmpera e revenimento. Para isso foram investigadas as variáveis de entrada do processo (diâmetro, velocidade, temperatura de revenimento e concentração do polímero) e as suas influências sobre o limite de resistência à tração, a estricção e a dureza do material. Os resultados revelaram que todas as variáveis consideradas têm influência significativa na obtenção das propriedades mecânicas e os modelos obtidos foram validados utilizando-se análise de variância (ANOVA). A regressão linear múltipla permitiu representar o processo adequadamente e a análise gráfica possibilitou visualizar o comportamento das múltiplas respostas.
\end{abstract}

Palavras-chave

Propriedades mecânicas. Planejamento de experimentos. Regressão linear múltipla. Têmpera e revenimento.

\section{Introdução}

A grande quantidade de informação gerada no mundo atual requer cada vez mais que as análises sejam criteriosas e bem-sucedidas. A parte mais importante da aplicação de métodos estatísticos é o planejamento dos experimentos que irão gerar os dados, os quais devem permitir a obtenção de conclusões válidas e significativas (PIMENTA, 2007).

Atualmente, devido à grande velocidade dos avanços científicos, se faz cada vez mais necessária a aplicação de métodos estatísticos para a otimização de processos industriais, pois esses repercutem na minimização de experimentos, na redução de custos para as empresas e, através da utilização de modelos estatísticos, é possível determinar as melhores condições de processamento, repercutindo diretamente na qualidade e produtividade.

As exigências do mercado de aços especiais, liderado pelos fabricantes de automóveis e de peças de reposição, obrigam as siderúrgicas a produzirem aços que atendam aos requisitos relacionados às propriedades mecânicas obtidas através de tratamento térmico de têmpera e revenimento.

Segundo Lima, Balestrassi e Paiva (2011), o delineamento de experimentos (DOE) é a metodologia mais adequada para estudar vários fatores de processo e a complexidade de suas interações, de forma a aumentar a probabilidade de solucionar problemas através de análises estatísticas. Essa metodologia é considerada poderosa para a melhoria da qualidade e produtividade, tendo sido nos últimos anos cada vez mais aplicada na indústria brasileira, principalmente pelas do segmento automobilístico (SILVA; SILVA, 2008).

A finalidade desta pesquisa é mostrar a aplicação das metodologias de planejamento de experimentos com análise em blocos e de regressão múltipla 
aplicadas ao estudo de um processo de têmpera e revenimento com múltiplas respostas. Nesse caso em especial, os métodos foram usados para auxiliar no desenvolvimento de uma modelagem estatística que viesse a substituir a maneira tradicional de ajuste das variáveis de entrada de um processo de tratamento térmico. Antes deste estudo, o ajuste inicial (setup) desse processo era realizado através do ensaio mecânico de uma amostra-piloto que, após passar por todas as fases de um tratamento térmico de têmpera e revenimento, era encaminhada para análise em laboratório. Os resultados obtidos nessa etapa eram usados para governar a regulagem do forno dentro da qual se fazia uma segunda amostrapiloto, que era novamente testada para confirmar que os ajustes do processo haviam sido suficientes para que o produto viesse a atingir as especificações, quando então a produção podia ser iniciada. Essa rotina operacional implicava em considerável tempo de análise e de espera, reduzindo, consequentemente, a produtividade do processo.

Esse cenário, comum em muitas siderúrgicas, tem sido a principal motivação para o desenvolvimento de modelos estatísticos que sejam úteis para a predição de resultados de propriedades mecânicas dos aços temperados e revenidos, visando com isto reduzir a quantidade de testes laboratoriais e o tempo de espera dos resultados e gerando, em consequência, maior produtividade e melhor nível de atendimento às especificações.

Este artigo está organizado da seguinte maneira: na seção 2 encontra-se a revisão bibliográfica abordando os conceitos de têmpera e revenimento, ensaios mecânicos e métodos estatísticos para planejamento e análise de experimentos. Na seção 3 são apresentados os materiais e métodos utilizados neste estudo. $\mathrm{Na}$ seção 4 são apresentados os resultados obtidos, acompanhados da discussão pertinente e, finalmente, na seção 5 são expostas as conclusões.

\section{Revisão bibliográfica}

\subsection{Têmpera e revenimento}

A têmpera está relacionada ao resfriamento brusco do aço após aquecimento à temperatura de austenitização e tem como objetivo a obtenção de uma microestrutura que proporcione propriedades mecânicas, tais como dureza e limite de resistência elevados, para aplicações específicas que requerem essa condição.

Segundo Callister Junior (2002), durante o estágio de resfriamento na têmpera a queda de temperatura promove transformações estruturais que acarretam o surgimento de tensões internas e por isso faz-se necessário a realização do revenimento, quando ocorre a transformação da martensita em martensita revenida. 0 processo de revenimento é realizado complementarmente à têmpera, sendo especialmente importante na fabricação de aços para molas. Ele consiste em aquecer o material temperado entre $250{ }^{\circ} \mathrm{C}$ e $650{ }^{\circ} \mathrm{C}$ por um determinado tempo, para aumentar a ductilidade e a elasticidade.

\subsection{Ensaios de limite de resistência à tração}

Segundo Mayers e Chawla (1982), em um teste de tração, o corpo de prova é fixado no cabeçote de uma máquina de ensaio que aplica um esforço que tende a alongá-lo até a ruptura, sendo medidas as deformações através de um aparelho chamado extensômetro. 0 ensaio é realizado num corpo de prova com dimensões padronizadas, para que os resultados obtidos possam ser comparados, reproduzidos e quantificados na própria máquina. Normalmente, o ensaio ocorre até a ruptura do material (o que o classifica como destrutivo) e permite medir a resistência do material e a deformação em função da tensão aplicada. Essa variação é extremamente útil para engenharia e é determinada pelo traçado da curva tensão-deformação. Acima de certo nível de tensão, os materiais começam a deformarem-se plasticamente até que haja a ruptura, ponto em que se obtém o limite de resistência à tração.

Nas indústrias siderúrgicas é muito utilizada a máquina de ensaio universal de tração e é comum que as unidades de força utilizadas sejam quilograma-força por milímetro quadrado $\left(\mathrm{kgf} / \mathrm{mm}^{2}\right)$ ou MegaPascal (MPa). As normas técnicas mais utilizadas para a execução de ensaios mecânicos são elaboradas pelas organizações ASTM (American Society for Testing and Materials) e ABNT (Associação Brasileira de Normas Técnicas).

\subsection{Ensaios de estricção}

Mayers e Chawla (1982) definem estricção como o atributo apresentado por certos materiais ao sofrerem grandes transformações plásticas quando submetidos à tensão de tração, antes de seu rompimento. Em corpos de prova de aço, a estricção é medida pela redução de área da seção transversal que ocorre antes da ruptura. A estricção é dada pelo quociente entre a variação da área transversal do corpo de prova (área inicial - área final) e o valor da área inicial da seção transversal. A estricção ou redução de área normalmente é expressa em porcentagem, mostrando quanto da área transversal da seção resistiva do corpo de prova foi reduzida após a aplicação da força (F) no ensaio de tração, conforme mostrado na Figura 1. 


\subsection{Ensaios de dureza}

Segundo Callister Junior (2002), a dureza é a medida da resistência de um metal à penetração. Os métodos mais comuns de determinar-se a dureza de um metal são o Brinell, o Vickers e o Rockwell. Nesta pesquisa será utilizado somente o método Brinell (HB).

As propriedades mecânicas dureza e limite de resistência à tração estão diretamente relacionadas. Em geral, quanto maior a dureza, maior será o limite de resistência e como nos aços frequentemente a dureza é proporcional à resistência ao desgaste e à durabilidade, ela é utilizada como uma forma de medir a resistência à abrasão.

A obtenção dos valores de dureza Brinell (HB), conforme mostrado na Figura 2, é feita dividindo-se a carga aplicada pela área de penetração. 0 penetrador de diâmetro (D) é uma esfera de aço temperado para

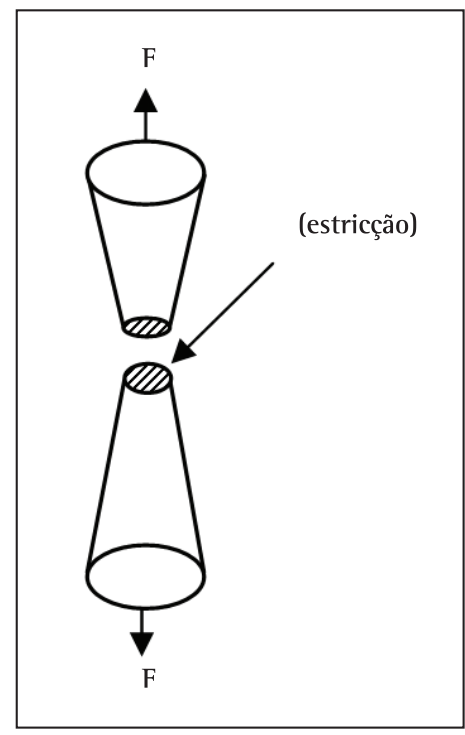

Figura 1. Redução de área após ruptura do corpo de prova (submetido à tração). Fonte: elaboração dos autores.

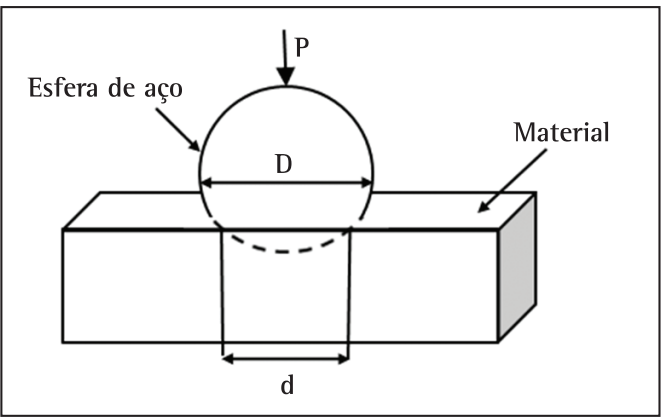

Figura 2. llustração do método de dureza Brinell (HB). Fonte: elaboração dos autores. materiais de dureza média ou baixa, ou de carboneto de tungstênio, para materiais de dureza elevada. A máquina de ensaio possui um microscópio óptico que faz a medição do diâmetro do círculo $(d, \mathrm{em} \mathrm{mm})$ que corresponde à projeção da calota esférica impressa na amostra. A dureza Brinell (HB) será dada pela carga aplicada ( $P$, em kgf) dividida pela área de impressão, conforme mostrado na Equação 1.

$$
H B=\frac{2 P}{\pi D\left(D-\sqrt{D^{2}}-d^{2}\right)}\left[\mathrm{kgf} / \mathrm{mm}^{2}\right]
$$

\subsection{Análise de experimentos}

No planejamento de experimentos, a primeira etapa é a escolha das variáveis (fatores) de entrada do processo que deverão ser investigadas. Em seguida, são escolhidas as variáveis de saída que serão monitoradas. Esses fatores de entrada poderão ser qualitativos ou quantitativos e as variáveis de saída, sempre que possível, deverão ser quantitativas, para proporcionar análises estatísticas mais precisas.

Segundo Rosa et al. (2009), Robin, Rosa e Silva (2010) e Souza et al. (2011), entre os métodos estatísticos mais apropriados para investigação de variáveis influentes em processos se encontra o planejamento ou delineamento de experimentos (DOE). Esse método é utilizado para definir fatores de entrada e variáveis de resposta, planejar os experimentos e estabelecer a ordem de experimentação para que se obtenham resultados com a maior precisão estatística ao menor custo possível.

Montgomery (2010) afirma que o planejamento fatorial é largamente utilizado em experimentos envolvendo diversos fatores nos quais seja necessário estudar o efeito de todos eles sobre uma ou mais respostas.

Segundo Barros Neto, Scarminio e Bruns (2007), para executar um planejamento fatorial deve-se especificar os níveis em que cada fator deverá ser estudado e o mais importante desses casos especiais é chamado de planejamento fatorial $2^{\mathrm{k}}$, que utiliza $k$ fatores de dois níveis cada. Nesse tipo de experimento, uma réplica completa requer $2 \times 2 \times 2 \times \ldots \times 2=2^{k}$ observações.

Segundo Montgomery (2010), a blocagem é uma técnica de projeto usada para melhorar a precisão da comparação entre fatores de interesse. Ela pode ser empregada em planejamentos fatoriais quando houver a necessidade de controlar a variabilidade proveniente de fontes perturbadoras conhecidas, que podem influir nos resultados. 


\subsection{Regressão linear múltipla e análise de variância (ANOVA)}

Segundo Benyounis e Olabi (2008), a técnica de regressão linear múltipla, quando utilizada complementarmente ao planejamento de experimentos, é muito eficiente para desenvolver modelos estatísticos que quantificam a influência das variáveis de entrada do processo para predição das variáveis de saída.

Segundo Montgomery e Runger (2003), a regressão linear múltipla é utilizada para situações que envolvam mais de um regressor. 0 modelo é dado pela Equação 2.

$Y=\beta_{0}+\beta_{1} x_{1}+\beta_{2} x_{2}+\ldots+\beta_{n} x_{n}+\varepsilon$

Nessa expressão, $Y$ representa a variável dependente, as variáveis independentes são representadas por $x_{n}, x_{2}, \ldots, x_{n}$ e $\varepsilon$ é o termo de erro aleatório. 0 termo linear é usado porque a equação é uma função linear dos parâmetros desconhecidos $\beta_{0}, \beta_{1}, \beta_{2}$ e $\beta_{n}$. Nesse modelo, o parâmetro $\beta_{0}$ é a interseção do plano, $\beta_{1}$, $\beta_{2 \mathrm{e}} \beta_{n}$ são os coeficientes parciais de regressão.

Os modelos que incluem efeitos de interação, segundo Montgomery e Runger (2003), também podem ser analisados pelo método de regressão linear múltipla. Uma interação entre duas variáveis pode ser representada por um termo cruzado, pois se admitirmos que $x_{3}=x_{1} x_{2}$ e $\beta_{3}=\beta_{12}$, então o modelo, incluindo termos de interação, ficará como apresentado na Equação 3.

$Y=\beta_{0}+\beta_{1} x_{1}+\beta_{2} x_{2}+\beta_{3} x_{3}+\ldots+\varepsilon$

Os termos quadráticos e de interação nos modelos podem produzir uma função formando uma curvatura (veja Equação 4), chamado modelo de segunda ordem. Dependendo dos valores dos coeficientes de regressão, o modelo de segunda ordem com interação é capaz de considerar uma ampla variedade de formas (MONTGOMERY; RUNGER, 2003).

$$
Y=\beta_{0}+\beta_{1} x_{1}+\beta_{2} x_{2}+\beta_{n} x_{1}^{2}+\beta_{n} x_{2}^{2}+\beta_{n} x_{3}+\ldots+\varepsilon
$$

0 coeficiente de determinação múltipla $\left(R^{2}\right)$ é uma medida da quantidade de redução na variabilidade de $Y$, obtida pelo uso dos regressores $x_{1}, x_{2}, \ldots, x_{k}$. 0 intervalo de variação deste coeficiente é de zero a um $\left(0 \leq R^{2} \leq 1\right)$. Se o valor de $R^{2}$ for próximo de um, isso significa que as diversas variáveis $x$ medidas são responsáveis quase que totalmente pela variabilidade de $Y$. Caso contrário, $\mathrm{R}^{2}$ apresentará um valor próximo à zero (MONTGOMERY; RUNGER, 2003).

Conforme Rosa et al. (2009) e Correia e Cardoza (2011), para analisar estatisticamente os resultados obtidos através de planejamento de experimentos, o método mais recomendado é a análise de variância (ANOVA), por meio da qual podem-se comparar três ou mais fatores e também testar-se a significância da regressão, fazendo uso do teste $F$ para comprovar quais os fatores e interações entre eles são realmente significantes no processo. A partir dos parâmetros calculados na ANOVA é possível criar um modelo estatístico através da técnica de regressão linear múltipla.

\subsection{Fatores importantes no ciclo de tratamento térmico}

A determinação dos fatores de entrada mais importantes dentro de um ciclo de tratamento térmico tem sido objeto de pesquisa por muitos autores.

Stein (2004) testou através de planejamento de experimentos as variáveis tempo de encharque (austenitização), temperatura de austenitização e temperatura de revenimento. Ele concluiu que a rápida austenitização, relacionada à velocidade de processamento, afeta sensivelmente as propriedades mecânicas e a microestrutura dos aços com teor de carbono acima de $0,4 \%$ na condição de temperado e revenido.

Rohatgi et al. (2005) utilizaram planejamento de experimentos para analisar a importância e a contribuição dos fatores fundição, fornecedor de lingote, testes de laboratório, volume e acabamento superficial na variável resposta limite de resistência à tração. Eles concluiram que através da determinação numérica dos efeitos dessas variáveis, a compreensão da dispersão nas propriedades foi reforçada e a reprodutibilidade da tração foi modelada estatisticamente. Para analisar os resultados, Rohatgi et al. (2005) utilizaram a ANOVA e constataram a influência de todos esses fatores sobre o limite de resistência à tração e sobre a dureza, sem entretanto haver encontrado interações significativas.

Ribeiro (2006), ao utilizar estatística para estudar as propriedades mecânicas do aço SAE 4140, constatou que essas propriedades são influenciadas pelos parâmetros de revenimento, em particular pela temperatura e tempo de tratamento térmico que estão relacionados à velocidade de processamento.

Mason e Young (2007) afirmaram que é possível obter bons resultados na modelagem estatística através da utilização de planejamento de experimentos em conjunto com a técnica de regressão linear múltipla e que modelos de regressão são atrativos porque poucas informações de processo são requeridas em seu desenvolvimento e implementação. 


\section{Materiais e métodos}

\subsection{Material utilizado nos ensaios}

0 material usado neste estudo foi o aço SAE 9254 trefilado a frio, utilizado para fabricação de molas de válvulas para o segmento automobilístico, com bitolas de diâmetro 2,00 mm e $6,50 \mathrm{~mm}$, submetido ao processo de têmpera e revenimento.

A análise química do material SAE 9254 usado no estudo é apresentada na Tabela 1.

\subsection{Processo de têmpera e revenimento usado na pesquisa}

Na Figura 3 é mostrado o fluxo básico do processo e as transformações de estrutura encontradas no tratamento de têmpera e revenimento de arames trefilados de aço com diâmetros de 2,00 mm e 6,50 mm.

$\mathrm{Na}$ zona de entrada do forno existem dez canais de engate por onde se inserem as bobinas de arame. Nessa primeira fase do processo, denominada entrada, o arame de aço possui estrutura bruta de trefilação (ferrita + perlita fina). Na segunda fase, denominada austenitização, o material passa por cinco zonas de temperatura, variando em torno de $900{ }^{\circ} \mathrm{C}$, momento em que ocorre a austenitização. $\mathrm{Na}$ terceira etapa do processo, o arame de aço é mergulhado em polímero líquido (meio de têmpera), momento em que a sua estrutura é transformada em martensita e, em seguida, é mergulhado em chumbo líquido, para ser revenido, com temperaturas variando de $400{ }^{\circ} \mathrm{C}$ a $480{ }^{\circ} \mathrm{C}$, com a finalidade de remoção das tensões superficiais e transformação da sua estrutura em martensita revenida. Na última fase do processo, o arame de aço é mergulhado em um tanque de óleo protetivo (antioxidante).

\subsection{Seleção dos fatores e organização experimental}

Os fatores investigados nesta pesquisa foram:

- Velocidade de passagem do arame dentro do forno (em m/s);

- Concentração do polímero, meio de têmpera (em \%);

- Temperatura do chumbo no revenimento (em $\left.{ }^{\circ} \mathrm{C}\right)$.
0 diâmetro do arame de aço também foi considerado um fator importante, pois existia a hipótese de que sua massa pudesse influenciar no resultado das propriedades mecânicas investigadas. Nesta pesquisa, porém, foi utilizada a metodologia de análise em blocos, ou seja, para o bloco 1 foram alocados os experimentos relacionados somente ao arame diâmetro 2,00 $\mathrm{mm}$ e para o bloco 2, os experimentos relacionados ao arame diâmetro $6,50 \mathrm{~mm}$, conforme mostrado na Tabela 2 .

Havia uma expectativa de que a variação simultânea dos fatores diâmetro do arame e velocidade iria gerar forte correlação entre eles pois, por limitações operacionais, quanto maior o diâmetro do arame, menor deveria ser a velocidade de processamento. Segundo Montgomery e Runger (2003), essa forte correlação entre fatores (de entrada) causa o fenômeno da multicolinearidade, que interfere diretamente nos resultados obtidos através do modelo estatístico, provocando grandes erros de predição. Duas

$\square$ Sequência do processo

Transformação da estrutura do material

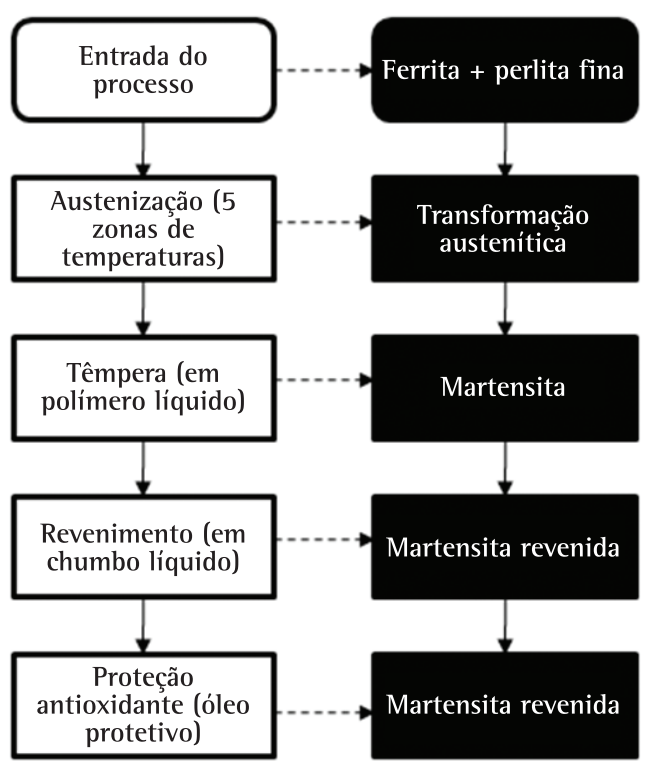

Figura 3. Fluxograma do processo de têmpera e revenimento e transformação da estrutura do material. Fonte: elaboração dos autores.

Tabela 1. Composição química (SAE 9254).

\begin{tabular}{cccccccccccccccc}
\hline $\begin{array}{c}\text { Elementos } \\
\text { químicos }\end{array}$ & $\mathrm{C}$ & $\mathrm{Mn}$ & $\mathrm{Si}$ & $\mathrm{P}$ & $\mathrm{S}$ & $\mathrm{Cr}$ & $\mathrm{Ni}$ & $\mathrm{Mo}$ & $\mathrm{Cu}$ & $\mathrm{Al}$ & $\mathrm{V}$ & \\
\hline$(\%)$ & 0,554 & 0,64 & 1,22 & 0,022 & 0,018 & 0,58 & 0,04 & 0,03 & 0,01 & 0,009 & 0,005 \\
\hline
\end{tabular}

Fonte: elaboração dos autores. 
possibilidades foram analisadas para neutralizar 0 efeito da multicolinearidade:

- Aplicar o método da análise de componentes principais (ACP), que utiliza combinações lineares, não correlacionadas entre si, das variáveis originais (PAIVA, 2006; MINGOTI, 2007). Para uma situação que envolvia múltiplas respostas, isso implicaria num modelo com maior complexidade e geraria maiores dificuldades de utilização prática na manufatura;

- Usar a blocagem para eliminar a correlação entre 0 fator diâmetro e o fator velocidade. Como a blocagem poderia ser facilmente operacionalizada, ela foi a estratégia adotada.

Os fatores velocidade, temperatura do chumbo e concentração do polímero foram experimentados através dos planejamentos fatoriais, utilizando-se a matriz $2^{3}$. Foi possível também testar os pontos centrais, representados por zero (0) na linha mais baixa da matriz, ou seja, além de testar os ajustes mínimos e máximos, também foram testados os pontos médios (pontos centrais) para cada fator e, dessa forma, a significância da curvatura, que irá definir a necessidade ou não de uma modelagem quadrática ou de segunda ordem para esse processo.

Para a realização do planejamento de experimentos foram utilizadas variáveis reduzidas $(\beta)$ ao invés de variáveis físicas (ajustes reais) dos fatores investigados, de forma a preservar os dados confidenciais da empresa financiadora da pesquisa. A redução das variáveis foi calculada segundo Montgomery e Runger (2003), utilizando o valor físico $(\alpha)$ que se quer testar subtraído da média $(\mu)$ entre os valores mínimo e máximo dos ajustes dos fatores. Dividiu-se o resultado pela metade da amplitude $(R)$ entre os valores mínimos e máximos dos ajustes dos fatores. Dessa forma, a

Tabela 2. Matriz fatorial $2^{3}$ usada para os blocos 1 e 2 .

\begin{tabular}{cccc}
\hline Experimentos & Velocidade & $\begin{array}{c}\text { Temperatura } \\
\text { chumbo }\end{array}$ & \% polimero \\
\hline 1 & - & - & - \\
2 & + & - & - \\
3 & - & + & - \\
4 & + & + & - \\
5 & - & - & + \\
6 & + & - & + \\
7 & - & + & + \\
8 & + & + & + \\
Ponto central & 0 & 0 & 0 \\
\hline
\end{tabular}

Fonte: elaboração dos autores. dimensionalidade das variáveis reduzidas ficou restrita ao intervalo [-1 a 1], que concentra os valores mínimo, médio e máximo, reduzidos dos ajustes dos fatores testados, conforme Equação 5 e Tabela 3.

$\beta=\frac{\alpha-\mu}{\frac{R}{2}}$

\section{Resultados e discussão}

\subsection{Sequência de experimentos e análise estatística}

Na experimentação foram realizadas inicialmente todas as réplicas relacionadas ao bloco 1 e, em seguida, as correspondentes ao bloco 2 . Seis réplicas foram utilizadas para cada condição experimental e outras seis para testar o ponto central para cada bloco. As replicações foram aleatorizadas e sequenciadas utilizando-se uma numeração de 1 a 9, correspondente à ordem de realização de cada experimento, para cada bloco individualmente. Essa sequência de experimentação é apresentada entre parênteses e em formato subscrito ao lado dos valores obtidos para as propriedades mecânicas, conforme mostrado nas Tabelas 4, 5 e 6 . É possível observar ainda que para cada condição experimental determinaram-se os valores das três propriedades mecânicas estudadas, correspondentes a cada réplica realizada.

A significância dos fatores foi testada a um nível de 95\% de confiança $(p<0,05)$. Essa análise foi realizada separadamente, para que pudesse ser verificada a significância dos fatores para cada uma das respostas das propriedades mecânicas estudadas, conforme mostrado nas Tabelas 7, 8 e 9.

Através do teste de significância realizado para a propriedade mecânica limite de resistência à tração (mostrado na Tabela 7), foi constatado que a curvatura é influente e os fatores significativos (onde $p<0,05$ ) são: diâmetro do arame (representado pela letra D e testado através de blocos), velocidade (representada pela letra A), temperatura do chumbo (representado pela letra B), concentração do polímero (representada pela letra $\mathrm{C}$ ), interações de segunda ordem entre velocidade e concentração do polímero, temperatura e

Tabela 3. Transformação de variáveis físicas para variáveis reduzidas.

\begin{tabular}{llc}
\hline \multicolumn{1}{c}{ Variáveis de entrada } & Valores (unidades físicas) & Valores (variáveis reduzidas) \\
\hline Velocidade $(\mathrm{m} / \mathrm{s})$ & Mínimo / Médio / Máximo & $-1 / 0 / 1$ \\
Temperatura do chumbo $\left({ }^{\circ} \mathrm{C}\right)$ & Mínimo / Médio / Máximo & $-1 / 0 / 1$ \\
Concentração do polímero $(\%)$ & Mínimo / Médio / Máximo & $-1 / 0 / 1$ \\
\hline
\end{tabular}

Fonte: elaboração dos autores. 
concentração do polímero e uma interação de terceira ordem entre velocidade, temperatura de chumbo e concentração do polímero. Também foi calculado o coeficiente de determinação de 99,34\% e o desvio padrão de 10,75 MPa.

Ao analisar-se o teste de significância para a propriedade mecânica estricção (mostrada na Tabela 8), é possivel constatar que a curvatura não é influente e os fatores influentes (onde $p<0,05$ ) são: diâmetro do arame (testado através de blocos), velocidade, temperatura do chumbo, concentração do polímero, interações de segunda ordem entre velocidade e temperatura de chumbo, velocidade e concentração do polímero, temperatura e concentração do polímero e uma interação de terceira ordem entre velocidade, temperatura do chumbo e concentração do polímero.

Tabela 4. Resultados de tração (MPa).

\begin{tabular}{|c|c|c|c|c|c|c|}
\hline Experimentos & Réplica 1 & Réplica 2 & Réplica 3 & Réplica 4 & Réplica 5 & Réplica 6 \\
\hline 1/Bloco 1 & $2.149_{(1)}$ & 2.148 & $2.146_{(2)}$ & 2.161 & 2.167 & $2.160_{(6)}$ \\
\hline 2/Bloco 1 & $2.157_{(4)}$ & $2.155_{(7)}$ & 2.157 & $2.151_{(7)}$ & $2.157_{\text {(4) }}$ & $2.157_{(2)}$ \\
\hline 3/Bloco 1 & 1.924 & 1.922 & 1.920 & 1.921 & $1.920_{(6)}$ & $1.918_{(4)}$ \\
\hline 4/Bloco 1 & $1.924_{(2)}$ & $1.924_{(8)}$ & $1.922_{(8)}$ & 1.943 (6) & $1.945_{(8)}$ & $1.945_{(5)}$ \\
\hline 5/Bloco 1 & $2.108_{(6)}$ & $2.106_{(5)}$ & $2.108_{(7)}$ & $2.104_{(2)}$ & 2.102 & $2.109_{(8)}$ \\
\hline 6/Bloco 1 & $2.136_{(5)}$ & 2.127 & 2.127 & $2.136_{(3)}$ & $2.134_{(3)}$ & 2.127 \\
\hline 7/Bloco 1 & $1.927_{(7)}$ & $1.926_{(2)}$ & $1.944_{(5)}$ & $1.935_{(4)}$ & $1.946_{(2)}$ & $1.947_{(7)}$ \\
\hline 8/Bloco 1 & 1.946 & $1.946_{(6)}$ & $1.946_{(6)}$ & 1.953 & 1.951 & 1.946 \\
\hline $\begin{array}{c}\text { Ponto central } \\
\text { bloco } 1\end{array}$ & 2.046 & $2.040_{(1)}$ & 2.041 & $2.049_{(9)}$ & $2.047_{(7)}$ & 2.053 \\
\hline 1/Bloco 2 & 1.968 & 1.974 & 1.962 & 1.971 & $1971_{(9)}$ & $1.974_{(5)}$ \\
\hline 2/Bloco 2 & $1.980_{(7)}$ & $1.976_{(4)}$ & 1.988 & $1.978_{(2)}$ & $1.980_{(3)}$ & $1.988_{(2)}$ \\
\hline 3/Bloco 2 & $1.771_{(3)}$ & $1.764_{(3)}$ & $1.763_{(7)}$ & $1.773_{(5)}$ & $1.771_{(5)}$ & $1.764_{(4)}$ \\
\hline 4/Bloco 2 & 1.796 & $1.784_{(2)}$ & 1.797 & 1.781 & $1.796_{(2)}$ & 1.784 \\
\hline 5/Bloco 2 & 1.949 & 1.963 (6) & 1.947 & 1.951 & 1.949 & $1.947_{(6)}$ \\
\hline 6/Bloco 2 & 1.992 & $1.980_{(5)}$ & 1.976 & $1.994_{(8)}$ & $1.980_{(7)}$ & $1.992_{(7)}$ \\
\hline 7/Bloco 2 & $1.760_{(2)}$ & $1.768_{(7)}$ & $1.766_{(5)}$ & $1.763_{(7)}$ & $1.766_{(6)}$ & 1.763 \\
\hline 8/Bloco 2 & $1.787_{(6)}$ & $1.793_{(8)}$ & $1.785_{(2)}$ & $1.784_{(6)}$ & 1.784 & 1.785 \\
\hline $\begin{array}{c}\text { Ponto central } \\
\text { bloco } 2\end{array}$ & $1.850_{(1)}$ & $1.847_{(9)}$ & $1.849_{(4)}$ & $1.848_{(3)}$ & $1.844_{(8)}$ & $1.846_{(3)}$ \\
\hline
\end{tabular}

Fonte: elaboração dos autores.

Tabela 5. Resultados de estricção em porcentagem (\%).

\begin{tabular}{|c|c|c|c|c|c|c|}
\hline Experimentos & Réplica 1 & Réplica 2 & Réplica 3 & Réplica 4 & Réplica 5 & Réplica 6 \\
\hline 1/Bloco 1 & $50_{(1)}$ & $51_{(9)}$ & $51_{(2)}$ & $50_{(8)}$ & $50_{(1)}$ & $50_{(6)}$ \\
\hline 2/Bloco 1 & $50_{(4)}$ & $50_{(7)}$ & $50_{(3)}$ & $50_{(7)}$ & $50_{(4)}$ & $50_{(2)}$ \\
\hline 3/Bloco 1 & $58_{(3)}$ & $58_{(3)}$ & 58 & $58_{(5)}$ & $58_{(6)}$ & $58_{(4)}$ \\
\hline 4/Bloco 1 & $58^{(2)}$ & $58_{(8)}$ & 58 & $56_{(6)}$ & $56_{(8)}$ & $56_{(5)}$ \\
\hline 5/Bloco 1 & $53_{(6)}$ & 53 & 53 & 53 & 53 & $53_{(8)}$ \\
\hline 6/Bloco 1 & $51_{(5)}$ & $52_{(4)}$ & $52_{(4)}$ & $51_{(3)}$ & $51_{(3)}$ & $52_{(3)}$ \\
\hline 7/Bloco 1 & $58_{(7)}$ & $58_{(2)}$ & $56_{(5)}$ & $58_{(4)}$ & $56_{(2)}$ & $56_{(7)}$ \\
\hline 8/Bloco 1 & $56_{(8)}$ & $56_{(6)}$ & $56_{(6)}$ & $55_{(1)}$ & $56_{(5)}$ & $56_{(9)}$ \\
\hline $\begin{array}{c}\text { Ponto central } \\
\text { bloco } 1\end{array}$ & $54_{(9)}$ & $54_{(1)}$ & $54_{(9)}$ & $54_{(9)}$ & $54_{(7)}$ & 53 \\
\hline 1/Bloco 2 & 42 & $41_{(1)}$ & $42_{(3)}$ & $42_{(4)}$ & $42_{(9)}$ & $41_{(5)}$ \\
\hline 2/Bloco 2 & $41_{(7)}$ & $41_{(4)}$ & $40_{(6)}$ & $41_{(2)}$ & $41_{(3)}$ & $40_{(2)}$ \\
\hline 3/Bloco 2 & 47 & $46_{(3)}$ & $46_{(7)}$ & $47_{(5)}$ & $47_{(5)}$ & $46_{(4)}$ \\
\hline 4/Bloco 2 & $44_{(8)}$ & $45_{(2)}$ & $44_{(8)}$ & $45_{(9)}$ & $44_{(2)}$ & $45_{(9)}$ \\
\hline 5/Bloco 2 & $56_{(5)}$ & $42_{(6)}$ & 56 & 56 & $56_{(4)}$ & $56_{(6)}$ \\
\hline 6/Bloco 2 & $40_{(4)}$ & $41_{(5)}$ & $41_{(9)}$ & $40_{(8)}$ & $41_{(7)}$ & $40_{(7)}$ \\
\hline 7/Bloco 2 & $46_{(2)}$ & $47_{(7)}$ & $47_{(5)}$ & $46_{(7)}$ & $47_{(6)}$ & $46_{(8)}$ \\
\hline 8/Bloco 2 & $44_{(6)}$ & $44_{(8)}$ & $45_{(2)}$ & $45_{(6)}$ & $45_{(1)}$ & 45 \\
\hline $\begin{array}{c}\text { Ponto central } \\
\text { bloco } 2\end{array}$ & $43_{(1)}$ & $43_{(9)}$ & $43_{(4)}$ & 43 & $43_{(8)}$ & $43_{(3)}$ \\
\hline
\end{tabular}

Fonte: elaboração dos autores. 
Também foi calculado o coeficiente de determinação de $85,27 \%$ e o desvio padrão de $2,40 \%$.

Analisando-se o teste de significância para a propriedade mecânica dureza (mostrada na Tabela 9), é possível afirmar que os fatores influentes (onde $p<0,05)$ são: diâmetro do arame (testado através de blocos), velocidade, temperatura do chumbo, concentração do polímero, interações de segunda ordem entre velocidade e concentração do polímero, temperatura e concentração do polímero

Tabela 6. Resultados de dureza (dureza Brinell).

\begin{tabular}{|c|c|c|c|c|c|c|}
\hline Experimentos & Réplica 1 & Réplica 2 & Réplica 3 & Réplica 4 & Réplica 5 & Réplica 6 \\
\hline 1/Bloco 1 & $608_{(1)}$ & $606_{(9)}$ & $606_{(2)}$ & $611_{(8)}$ & $611_{(1)}$ & $611_{(6)}$ \\
\hline 2/Bloco 1 & $608_{(4)}$ & $608_{(7)}$ & $608_{(3)}$ & $608_{(7)}$ & $608_{(4)}$ & $608_{(2)}$ \\
\hline 3/Bloco 1 & $544_{(3)}$ & $542_{(3)}$ & $542_{(1)}$ & $542_{(5)}$ & $542_{(6)}$ & $542_{(4)}$ \\
\hline 4/Bloco 1 & $544_{(2)}$ & $544_{(8)}$ & $542_{(8)}$ & $550_{(6)}$ & $550_{(8)}$ & $550_{(5)}$ \\
\hline 5/Bloco 1 & $594_{(6)}$ & $594_{(5)}$ & $594_{(7)}$ & $594_{(2)}$ & 594 (9) & $594_{(8)}$ \\
\hline 6/Bloco 1 & $603_{(5)}$ & $600_{(4)}$ & $600_{(4)}$ & 603 & $603_{(3)}$ & $600_{(3)}$ \\
\hline 7/Bloco 1 & $544_{(7)}$ & $544_{(2)}$ & $550_{(5)}$ & $547_{(4)}$ & $550_{(2)}$ & $550_{(7)}$ \\
\hline 8/Bloco 1 & $550_{(8)}$ & $550_{(6)}$ & $550_{(6)}$ & $553_{(1)}$ & $550_{(5)}$ & $550_{(9)}$ \\
\hline $\begin{array}{l}\text { Ponto central } \\
\text { bloco } 1\end{array}$ & 578 (9) & $575_{(1)}$ & $578_{(9)}$ & $578_{(9)}$ & $578_{(7)}$ & 581 \\
\hline 1/Bloco 2 & 556 (9) & $558_{(1)}$ & $556_{(3)}$ & $556_{(4)}$ & $556_{(9)}$ & $558_{(5)}$ \\
\hline 2/Bloco 2 & $558_{(7)}$ & $558_{(4)}$ & $561_{(6)}$ & $558_{(2)}$ & $558_{(3)}$ & $561_{(2)}$ \\
\hline 3/Bloco 2 & $500_{(3)}$ & 497 & $497_{(7)}$ & $500_{(5)}$ & $500_{(5)}$ & 497 \\
\hline 4/Bloco 2 & $508_{(8)}$ & $503_{(2)}$ & $508_{(8)}$ & 503 & $508_{(2)}$ & 503 (9) \\
\hline 5/Bloco 2 & $550_{(5)}$ & $556_{(6)}$ & $550_{(1)}$ & $550_{(1)}$ & $550_{(4)}$ & $550_{(6)}$ \\
\hline 6/Bloco 2 & $564_{(4)}$ & $558_{(5)}$ & $558_{(9)}$ & $564_{(8)}$ & $558_{(7)}$ & $564_{(7)}$ \\
\hline 7/Bloco 2 & $4977_{(2)}$ & $500_{(7)}$ & $500_{(5)}$ & $497_{(7)}$ & $500_{(6)}$ & $497_{(8)}$ \\
\hline 8/Bloco 2 & $506_{(6)}$ & $506_{(8)}$ & $503_{(2)}$ & $503_{(6)}$ & 503 & 503 (1) \\
\hline $\begin{array}{l}\text { Ponto central } \\
\text { bloco } 2\end{array}$ & $522_{(1)}$ & $522_{(9)}$ & $522_{(4)}$ & $522_{(3)}$ & $519_{(8)}$ & $522_{(3)}$ \\
\hline
\end{tabular}

Fonte: elaboração dos autores.

Tabela 7. Teste de significância para limite de resistência (em MPa).

\begin{tabular}{|c|c|c|c|c|}
\hline Termos & Efeito & Coeficiente & $\mathrm{T}$ & $\mathrm{p}$ \\
\hline Constante & & $1.955,29$ & $1.782,89$ & 0,000 \\
\hline (D) & 165,62 & 82,81 & 80,09 & 0,000 \\
\hline (A) & 17,42 & 8,71 & 7,94 & 0,000 \\
\hline (B) & $-198,54$ & $-99,27$ & $-90,52$ & 0,000 \\
\hline (C) & $-8,04$ & $-4,02$ & $-3,67$ & 0,000 \\
\hline (A)(B) & $-0,54$ & $-0,27$ & $-0,25$ & 0,805 \\
\hline$(\mathrm{A})(\mathrm{C})$ & 5,62 & 2,81 & 2,56 & 0,012 \\
\hline (B)(C) & 14,08 & 7,04 & 6,42 & 0,000 \\
\hline$(\mathrm{A})(\mathrm{B})(\mathrm{C})$ & $-6,25$ & $-3,13$ & $-2,85$ & 0,005 \\
\hline Curvatura & & $-8,63$ & $-2,62$ & 0,010 \\
\hline
\end{tabular}

Fonte: elaboração dos autores.

Tabela 8. Teste de significância para estricção (em porcentagem).

\begin{tabular}{crrrr}
\hline Termos & Efeito & Coeficiente & T & p \\
\hline Constante & & 49,458 & 201,94 & 0,000 \\
(D) & 9,426 & 4,713 & 201,94 & 0,000 \\
(A) & $-2,750$ & $-1,375$ & $-5,61$ & 0,000 \\
(B) & 3,583 & 1,792 & 7,32 & 0,000 \\
(C) & 1,750 & 0,875 & 3,57 & 0,001 \\
(A)(B) & 1,250 & 0,625 & 2,55 & 0,012 \\
(A)(C) & $-1,667$ & $-0,833$ & $-3,40$ & 0,001 \\
(B)(C) & $-2,250$ & $-1,125$ & $-4,59$ & 0,000 \\
(A)(B)(C) & 1,667 & 0,833 & 3,40 & 0,001 \\
Curvatura & & $-1,042$ & $-1,42$ & 0,159 \\
\hline
\end{tabular}


Tabela 9. Teste de significância para dureza (em HB).

\begin{tabular}{|c|c|c|c|c|}
\hline Termos & Efeito & Coeficiente & $\mathrm{T}$ & $\mathrm{p}$ \\
\hline Constante & & 552,09 & $1.650,05$ & 0,000 \\
\hline (D) & 46,86 & 23,43 & 74,26 & 0,000 \\
\hline (A) & 4,85 & 2,43 & 7,25 & 0,000 \\
\hline (B) & $-55,81$ & $-27,91$ & $-83,40$ & 0,000 \\
\hline (C) & $-2,19$ & $-1,09$ & $-3,27$ & 0,001 \\
\hline (A)(B) & 0,10 & 0,05 & 0,16 & 0,877 \\
\hline$(\mathrm{A})(\mathrm{C})$ & 1,65 & 0,82 & 2,46 & 0,016 \\
\hline (B)(C) & 4,06 & 2,03 & 6,07 & 0,000 \\
\hline$(\mathrm{A})(\mathrm{B})(\mathrm{C})$ & $-2,35$ & $-1,18$ & $-3,52$ & 0,001 \\
\hline Curvatura & & $-2,34$ & $-2,33$ & 0,022 \\
\hline
\end{tabular}

Fonte: elaboração dos autores.

e uma interação de terceira ordem entre velocidade, temperatura do chumbo e concentração do polímero. A curvatura nesse caso é influente, o coeficiente de determinação resultou em 99,23\% e o desvio padrão em 3,28 Brinell (HB).

Analisando-se os valores do coeficiente de determinação de todos os testes de significância e conforme critérios estabelecidos por Montgomery (2010), é possível afirmar que em todas as situações a correlação poderá ser considerada forte, pois as diversas variáveis independentes medidas são responsáveis quase que totalmente pela variabilidade da variável resposta. Os valores dos desvios padrão foram considerados pequenos, pois as próprias variações de repetitividade e reprodutibilidade dos instrumentos de medição utilizados são superiores ao valor dos desvios.

\subsection{Modelagem estatística para as múltiplas respostas}

Utilizando os coeficientes calculados através do teste de significância foi possível construir modelos estatísticos que representam a relação entre as variáveis de entrada do processo (fatores) e as variáveis de saída (propriedades mecânicas). Esses modelos estatísticos estão definidos nas Equações 6, 7 e 8.

$$
\begin{aligned}
& \mathrm{LR}=1955,29+82,81(\mathrm{D})+8,71(\mathrm{~A})-99,27(\mathrm{~B}) \\
& -4,02(\mathrm{C})+2,81(\mathrm{~A})(\mathrm{C})+7,04(\mathrm{~B})(\mathrm{C})-3,13(\mathrm{~A})(\mathrm{B})(\mathrm{C}) \\
& \mathrm{E}=49,458+4,713(\mathrm{D})-1,375(\mathrm{~A}) \\
& +1,792(\mathrm{~B})+0,875(\mathrm{C})+0,625(\mathrm{~A})(\mathrm{B}) \\
& -0,833(\mathrm{~A})(\mathrm{C})-1,125(\mathrm{~B})(\mathrm{C})+0,833(\mathrm{~A})(\mathrm{B})(\mathrm{C}) \\
& \mathrm{D}=552,09+23,43(\mathrm{D})+2,43(\mathrm{~A})-27,91(\mathrm{~B}) \\
& -1,09(\mathrm{C})+0,82(\mathrm{~A})(\mathrm{C})+2,03(\mathrm{~B})(\mathrm{C})-1,18(\mathrm{~A})(\mathrm{B})(\mathrm{C})
\end{aligned}
$$

Onde:

- LR: corresponde à variável resposta limite de resistência à tração;

- E: corresponde à variável resposta estricção;

- D: corresponde à variável resposta dureza.

Além das análises de significância utilizadas para cálculo de coeficientes para os modelos e para calcular a influência dos fatores, também foram realizadas análises de variâncias para validar os modelos estatísticos para cada variável resposta. Essa validação tornou-se necessária devido à constatação de a influência da curvatura das propriedades mecânicas limite de resistência à tração e dureza ser significante e, conforme mostrado nas Tabelas 10, 11 e 12, foi possivel observar que os modelos lineares e modelos lineares com termos de interação possuem maior influência, com valores de significância $(p)$ inferiores aos modelos de segunda ordem (quadráticos).

Com isso, utilizando critérios baseados nas análises de variância, os modelos lineares, incluindo-se os termos de interação mostrados nas Equações 6, 7 e 8 (já apresentadas), foram escolhidos para representar 0 processo de têmpera e revenimento em suas múltiplas respostas.

\subsection{Análise gráfica da significância dos efeitos dos fatores}

A análise gráfica revela que embora todos os fatores sejam significativos para a obtenção das propriedades mecânicas, a temperatura do chumbo (fator B) e a velocidade (fator A) destacam-se em todos os casos, veja Figuras 4, 5 e 6 . No eixo horizontal plotam-se os valores dos efeitos padronizados através da estatística T para cada fator e, no eixo vertical, estão representados os fatores. Em todos os casos, o valor crítico é $T=1,98$, e sempre que o valor da estatística $\mathrm{T}$ de cada efeito supera esse valor (crítico) para 95\% de confiança, ele é dito significativo. 


\subsection{Média dos efeitos dos fatores sobre as}

\section{respostas}

A média dos efeitos em relação à resposta limite de resistência à tração é apresentada na Figura 7.

Analisando-se essa figura é possivel constatar que os ajustes dos fatores que proporcionam aumento no limite de resistência à tração são:
- Diâmetro do arame (blocos) no nível 1;

- Velocidade no nível 1;

- Temperatura do chumbo no nível -1;

- Concentração do polímero no nível -1.

A média dos efeitos em relação à resposta estricção é apresentada na Figura 8.

Em relação à propriedade mecânica estricção (redução de área), os ajustes dos fatores que

Tabela 10. Análise de variância para validação do modelo para limite de resistência à tração.

\begin{tabular}{lcccrc}
\hline Modelos de regressão & Grau de liberdade & Soma dos quadrados & Quadrados médios & $\mathrm{F}$ & $\mathrm{p}$ \\
\hline Linear & 4 & 1.695 .579 & 423.895 & $3.646,45$ & 0,000 \\
Quadrática & 1 & 794 & 794 & 6,83 & 0,010 \\
Interação & 6 & 6.620 & 1.103 & 9,49 & 0,000 \\
Erro residual & 96 & 11.160 & 11.160 & & \\
\hline
\end{tabular}

Fonte: elaboração dos autores.

Tabela 11. Análise de variância para validação do modelo para estricção.

\begin{tabular}{lccrrr}
\hline Modelos de regressão & Grau de liberdade & Soma dos quadrados & Quadrados médios & $\mathrm{F}$ & $\mathrm{p}$ \\
\hline Linear & 4 & $2.962,06$ & 740,516 & 174,52 & 0,000 \\
Quadrática & 1 & 11,57 & 11,574 & 0,102 \\
Interação & 6 & 449,33 & 74,889 & 17,65 \\
Erro residual & 96 & 407,35 & 4,243 & 0,000 \\
\hline
\end{tabular}

Fonte: elaboração dos autores.

Tabela 12. Análise de variância para validação do modelo para dureza.

\begin{tabular}{lccccc}
\hline Modelos de regressão & Grau de liberdade & Soma dos quadrados & Quadrados médios & F & p \\
\hline Linear & 4 & 134.709 & $33.677,2$ & 58,6 & 5,000 \\
Quadrática & 1 & 59 & 88,5 & 0,027 \\
lnteração & 6 & 531 & 11,6 & 7,61 \\
Erro residual & 96 & 1.116 & 0,000 \\
\hline
\end{tabular}

Fonte: elaboração dos autores.

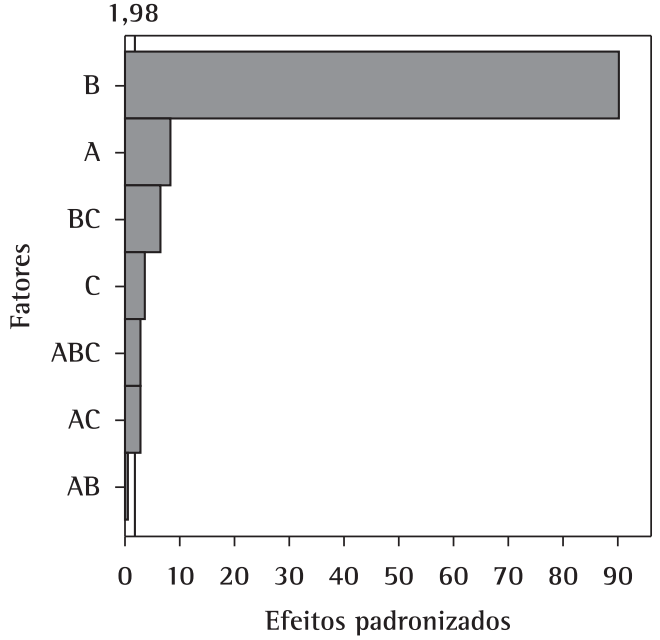

\begin{tabular}{|ll|}
\hline Fator & Nome \\
A & Velocidade \\
B & Temperatura \\
C & Polímero \\
\hline
\end{tabular}

Figura 4. Influência dos fatores na resposta limite de resistência à tração. Fonte: elaboração dos autores.

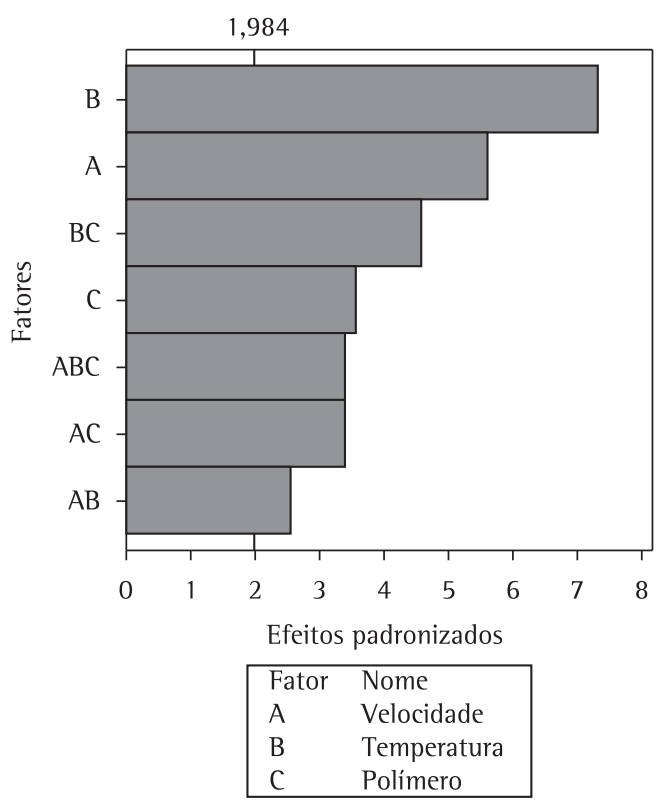

Figura 5. Influência dos fatores na resposta estricção. Fonte: elaboração dos autores. 


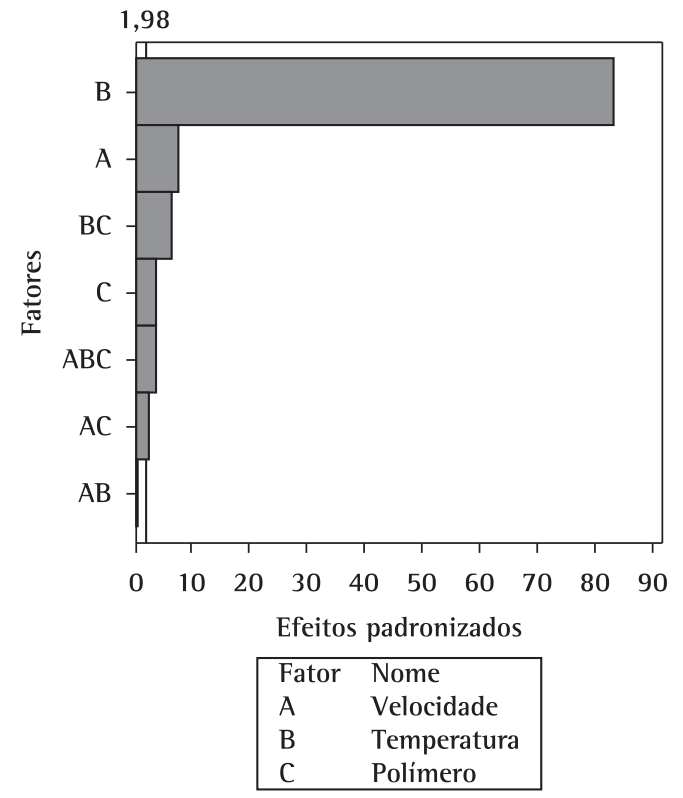

Figura 6. Influência dos fatores na resposta dureza. Fonte: elaboração dos autores.

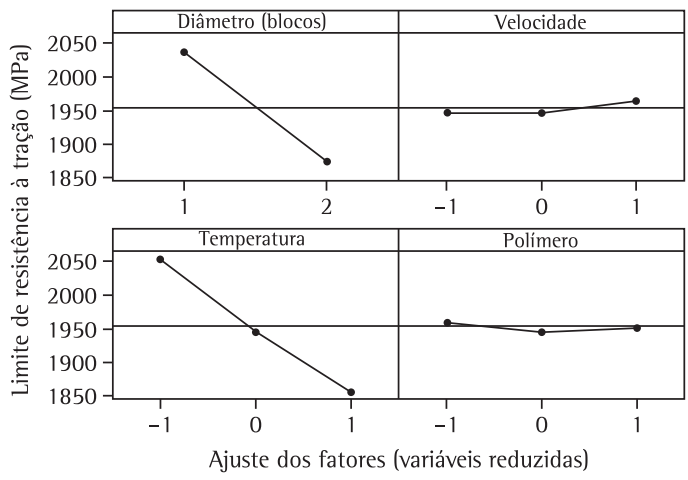

Figura 7. Média dos efeitos sobre o limite de resistência à tração. Fonte: elaboração dos autores.

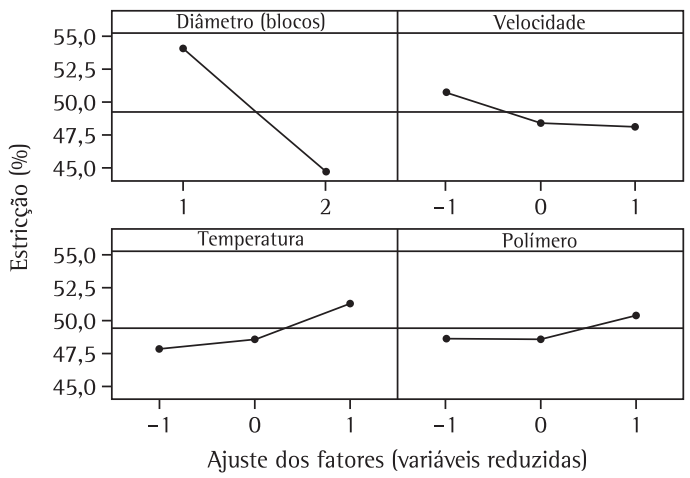

Figura 8. Média dos efeitos sobre a estricção. Fonte: elaboração dos autores. proporcionam maiores valores, conforme mostrado nessa figura, são:

- Diâmetro do arame (blocos) no nível 1;

- Velocidade no nível -1;

- Temperatura do chumbo no nível 1 ;

- Concentração do polímero no nível 1.

A média dos efeitos em relação à resposta dureza é apresentada na Figura 9.

Analisando-se essa figura percebe-se que os ajustes dos fatores que proporcionam maiores valores de dureza são:

- Diâmetro do arame (blocos) nível 1;

- Velocidade no nível 1;

- Temperatura do chumbo nível -1;

- Concentração do polímero nível -1.

A análise dos gráficos nas Figuras 7, 8 e 9 orienta 0 ajuste do processo de forma a atender às especificações das propriedades mecânicas.

\subsection{Análise gráfica do comportamento das múltiplas respostas}

Analisando-se a Figura 10 é possível observar que existe relação entre as múltiplas respostas (propriedades mecânicas), isto é, determinando-se valores para as propriedades mecânicas dureza e estricção é possivel estimar a faixa de valores correspondentes para a propriedade mecânica limite de resistência à tração. Por exemplo, para o arame com diâmetro de 2,00 mm, se foi obtida uma dureza de 570 HB e uma estriç̧ão de $51 \%$, é de se prever que o limite de resistência à tração esteja entre 2.050 MPa e 2.100 MPa.

Da maneira análoga, é possível estimar valores de propriedades mecânicas para o arame de diâmetro $6,50 \mathrm{~mm}$. Por exemplo, para uma dureza de $535 \mathrm{HB}$ e estricção de $46 \%$, é de se esperar que o limite de

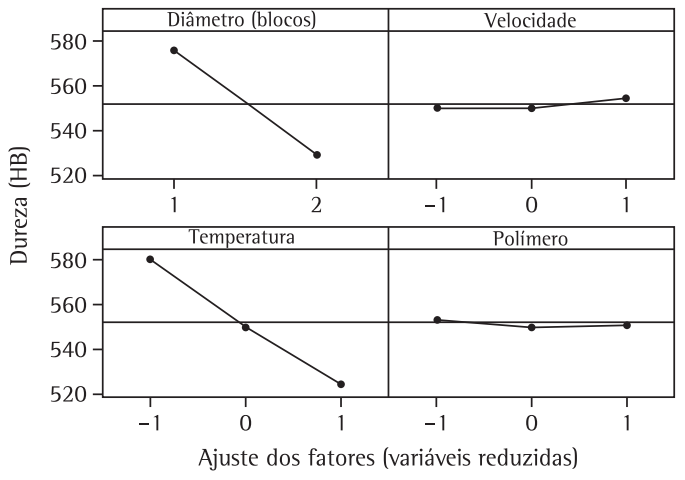

Figura 9. Média dos efeitos sobre a dureza (HB). Fonte: elaboração dos autores. 


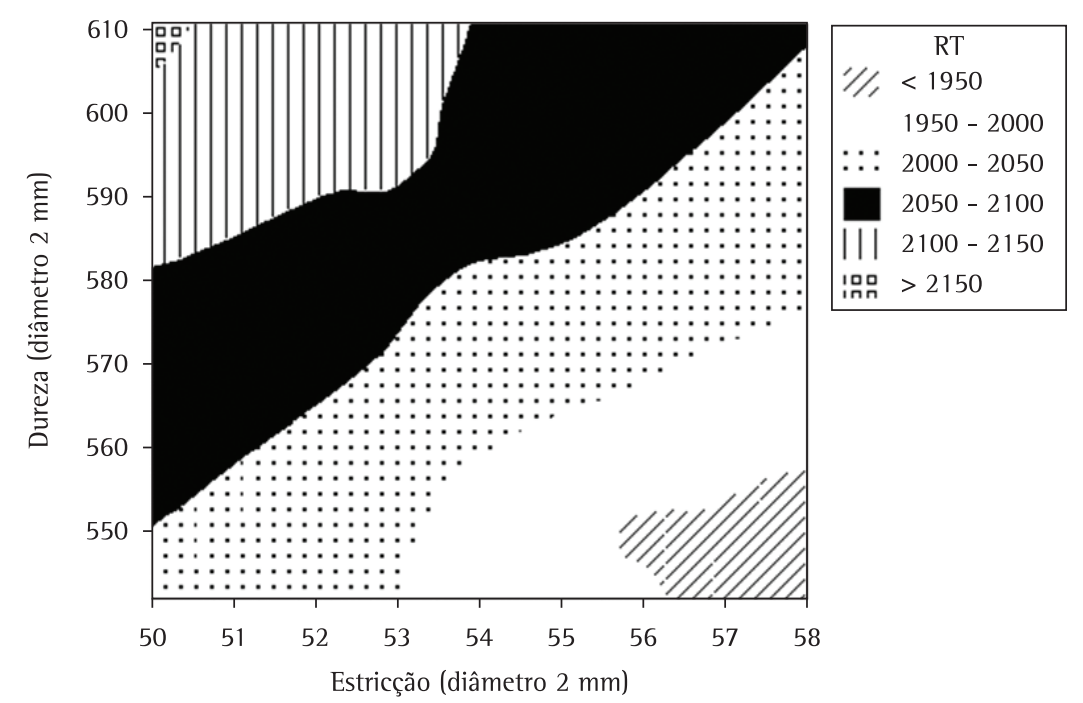

Figura 10. Comportamento das múltiplas respostas (diâmetro 2,00 mm). Fonte: elaboração dos autores.

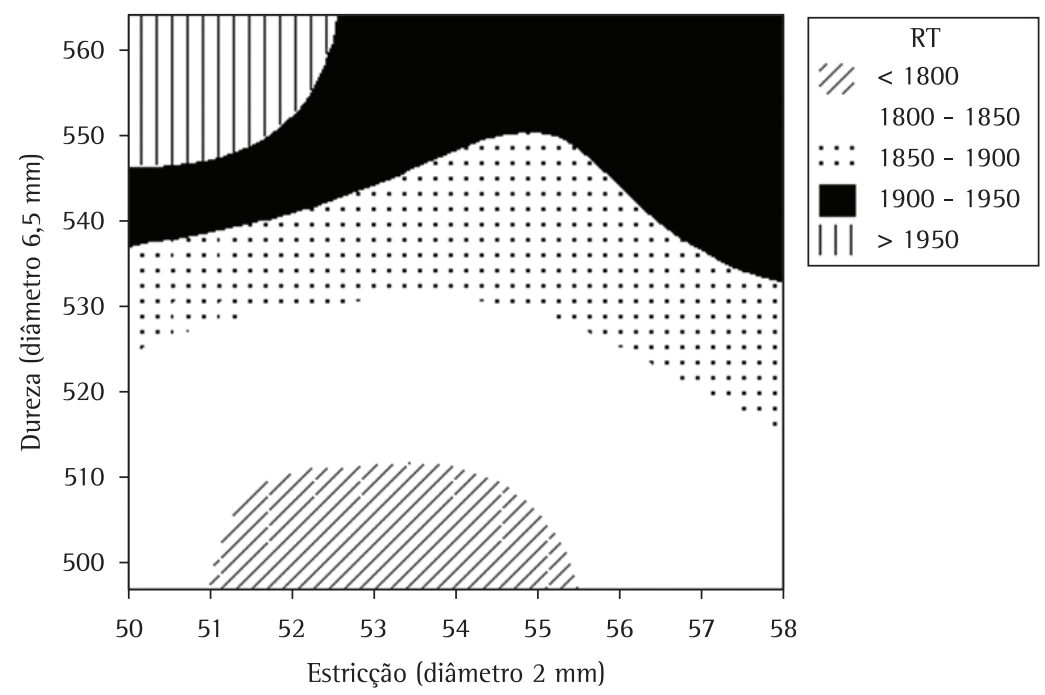

Figura 11. Comportamento das múltiplas respostas (diâmetro $6,50 \mathrm{~mm}$ ). Fonte: elaboração dos autores.

resistência à tração esteja entre $1.850 \mathrm{MPa}$ e $1.900 \mathrm{MPa}$ (ver Figura 11). Dessa forma, é possivel escolher as melhores regiões das propriedades mecânicas, de modo a obter resultados que atendam às especificações $\mathrm{e}$ com um direcionamento para os melhores ajustes dos fatores.

\section{Conclusões}

A aplicação da metodologia de planejamento de experimentos com análise em blocos conduzida para o tratamento térmico de têmpera e revenimento de arames de aço SAE 9254 trefilado com diâmetros de 2,00 $\mathrm{mm}$ e $6,50 \mathrm{~mm}$ proporcionou a compreensão ampla da influência dos fatores nas propriedades mecânicas limite de resistência à tração, estricção e dureza.

Os resultados revelaram, através do teste de significância, que os fatores diâmetro, velocidade, temperatura de revenimento e concentração do polímero têm influência significativa nas propriedades mecânicas estudadas.

A regressão linear múltipla se mostrou eficiente para representar o processo adequadamente e a análise 
gráfica possibilitou visualizar o comportamento das múltiplas respostas.

\section{Referências}

BARROS NETO, B.; SCARMINIO, I. S.; BRUNS, R. E. Como fazer experimentos: Pesquisa e Desenvolvimento na Ciência e na Indústria. 3. ed. Campinas: UNICAMP, 2007. 480 p.

BENYOUNIS, K. Y.; OLABI, A. G. Optimization of different welding processes using statistical and numerical approaches - A reference guide. Advances in Engineering Software, v. 39, n. 6, p. 483-496, 2008. http://dx.doi. org/10.1016/j.advengsoft.2007.03.012

CALLISTER JUNIOR, W. D. Uma introdução a engenharia e a ciências dos materiais. 5. ed. São Paulo: LTC, 2002. 589 p.

CORREIA, E. A. S.; CARDOZA, J. A. S. Planejamento de experimentos no processo produtivo utilizando o método Taguchi. Gestão da Produção, Operações e Sistemas, n. 1, p. 55-66, 2011.

LIMA, V. B. S.; BALESTRASSI, P. P.; PAIVA, A. P. Otimização do desempenho de amplificadores de radio frequência banda larga: uma abordagem experimental. Produção, v. 21 , n. 1, p. 118-131, 2011.

MASON, L. R.; YOUNG, C. J. Monitor your industrial processes. Quality Progress, v. 39, n. 4, p. 89-90, 2007.

MAYERS, A. M.; CHAWLA, K. K. Princípios de metalurgia mecânica. 2. ed. São Paulo: Edgard Blucher, 1982. 422 p.

MINGOTI, S. A. Análise de dados através de métodos de estatística multivariada, uma abordagem aplicada. Belo Horizonte: UFMG, 2007. 295 p.

MONTGOMERY, C. D. Design and analysis of experiments. 7th ed. John Wiley \& Sons, 2010. 203 p.

MONTGOMERY, D. C.; RUNGER, G. C. Estatística aplicada e probabilidade para engenheiros. 2. ed. São Paulo: LTC, 2003. p. 230-320
PAIVA, A. P. Metodologia de superfície de resposta e análise de componentes principais em otimização de processos de manufatura com múltiplas respostas correlacionadas. 2006. 229 f. Tese (Doutorado)Universidade Federal de Itajubá, Itajubá, 2006.

PIMENTA, C. D. et al. Planejamento de experimentos (doe) aplicado no processo de têmpera e revenimento de arames de aço SAE 9254. Jânus, v. 5, n. 8, p. 119-139, 2007.

RIBEIRO, L. P. P. G. Caracterização das Propriedades Mecânicas do Aço SAE 4140 após Diferentes Tratamentos de Têmpera e Revenido. 2006. 100 f. Dissertação (Mestrado)-Pontifícia Universidade Católica do Rio de Janeiro, Rio de Janeiro, 2006.

ROBIN; A.; ROSA, J. L.; SILVA, M. B. Electrodeposition and characterization of $\mathrm{Cu}-\mathrm{Nb}$ composite coatings. Surface \& Coatings Technology, v. 205, p. 2152-2159, 2010. http://dx.doi.org/10.1016/j.surfcoat.2010.08.124

ROHATGl, P. K. et al. Scatter and statistical analysis of tensile properties of cast SiC reinforced A359 alloys. Materials Science and Engineering, v. 398, n. 1-2, p. 1-14, 2005. http://dx.doi.org/10.1016/j.msea.2005.03.035

ROSA, J. L. et al. Electrodeposition of copper on titanium wires: Taguchi experimental design approach. Journal of Materials Processing Technology, v. 209, p. 1181-1188, 2009.http://dx.doi.org/10.1016/j. jmatprotec.2008.03.021

SILVA H, A.; SILVA M. B. Aplicação de um projeto de experimentos (DOE) na soldagem de tubos de zircaloy-4. Produção \& Engenharia, v. 1, n. 1, p. 41-52, 2008.

SOUZA, H. J. C. et al. Optimization Molecular assay optimized by Taguchi experimental design method for venous thromboembolism investigation. Molecular and Cellular Probes, v. 25, p. 231-237, 2011. PMid: 21867748.

STEIN, C. R. Efeito da rápida austenitização sobre as propriedades mecânicas de um aço C-Mn. 2004. $73 \mathrm{f}$. Dissertação (Mestrado)-Universidade Federal de Ouro Preto, Ouro Preto, 2004.

\section{Design of block experiments applied to the mechanical properties of steel wires for springs}

\section{Abstract}

The purpose of this article is to demonstrate an application of the design of block experiments via analysis and multiple linear regression in the investigation of a steel thermal treatment process with multiple responses. The study aimed to design statistical models to predict the mechanical properties in SAE 9254 draw steel wires, with diameters of $2.00 \mathrm{~mm}$ and $6.50 \mathrm{~mm}$, after quench hardening and tempering. For this purpose, process input variables (wire diameter, processing speed, tempering temperature and polymer concentration) were investigated regarding their influence on the material tensile strength, yield point and hardness. The results revealed that the mechanical properties of the steel wire are significantly influenced by the selected variables, and analysis of variance (ANOVA) was employed to validate the design of the statistical models. Multiple linear regression allowed for an appropriate representation of the process, and graphical analysis was found to be very useful in displaying the behavior of the multiple responses.

\section{Keywords}

Mechanical properties. Design of experiments. Multiple linear regression. Quench hardening and tempering. 